\title{
Low Area, Low Power and Wide Bandwidth Operational Amplifier by 130nm CMOS Technology
}

\author{
Chintan Suman Verma ${ }^{1}$, Dr. R. H. Talwekar ${ }^{2}$ \\ ${ }^{1}$ Electronics and Telecommunication, SSTC-SSGI, (C.G.), Bhilai, India \\ ${ }^{2}$ Vice-Principal and Prof. of SSITM, SSITM-SSGI, (C.G.), Bhilai, India
}

\begin{abstract}
A high performance two-stage Operational Amplifier is presented in this paper having the specifications of less Area and wide bandwidth which is operated on low power i.e. $1.3 \mathrm{~V}$ supply. Miller capacitor with resistor is used for compensation which also significantly improves the bandwidth. Design of Operational Amplifier is demonstrated with only CMOS transistors. The Amplifier exhibits a gain of $59 \mathrm{~dB}, 1.25 \mathrm{MHz} 3 \mathrm{~dB}$ bandwidth, $605 \mathrm{MHz}$ Unity gain bandwidth, $269 \mathrm{~dB}$ CMRR, $106 \mathrm{~dB}$ PSRR, $1.6 \mathrm{~V} / \mu \mathrm{s}$ Slew rate with the power dissipation of $0.43 \mathrm{~mW}$.
\end{abstract}

Keywords: Op-amp; low power; less area; two-stage; CMRR.

\section{Introduction}

The Mixed signal applications increased with ICs contains digital and analog parts, along with technology scaling motivates for development of a device which can be used for both. Operational Amplifier (commonly referred as Op-amp) attracts our own side for this type of design. It is the basic element of many analog integrated circuit designs beyond to this it uses vastly different levels of complexity to comprehend functions ranging from dc bias generation to high speed amplification or filtering. It is not only limited for amplifying the signals but it can perform large number of operation also such as mathematical operation, A/D and D/A conversion, clipping, clamping, zero-crossing detection, pulse generatoretc. In the era of advance technology researchers move to work with low power supplywhich is very useful for portable devices such as laptops, tablets, mobiles, pacemaker etc. so that it can operate with low supply as well as power dissipation is can be easily managed which is the major issue for designing any system. High speed device required for fast processing applications, considering this parameter a method is proposed for obtaining high bandwidth in this research work. Miller compensation with resistor is used for maintaining stability for this Op-amp.In this technique a resistor is connected in series with capacitor. This resistor and capacitor takes large die area but in the today's technology the demand of low area for high chip density. In this work both these components are replaced with MOS transistors which takes very small area compared to this.Along with this current source is used in the conventional Op-amp to maintain transistors in proper region. This is also to be designed with MOS transistors. So finally in this research work less area, low power and wide bandwidth Op-amp is presented.

\section{Used Compensation Technique}

For designing any analog circuit compensation is required for maintaining stability. In this proposed Op-amp miller compensation with resistor is used for maintaining stability. This is also to be referred as nulling resistor compensation technique. In this technique a resistor is connected in series with miller capacitor across the second stage which provides more stability compared to miller compensation technique. This technique also improves the $3 \mathrm{~dB}$ bandwidth. The configuration of this technique is shown below.

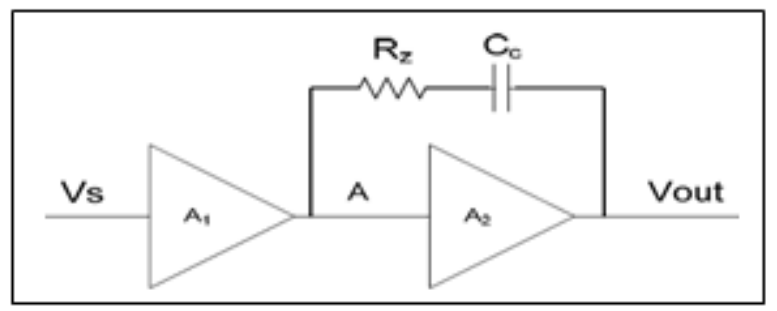

Figure 1: Nulling resistor compensation

As in the previous Op-amp miller compensation technique is used which splits the pole i.e. dominant pole which is nearest to origin and non-dominant pole which is far away from origin. As well as it generates the RHP zero which affects the stability. This RHP zero is generating due to feedforward current flow in the feedforward path. This feedforward path is created due to the direct connection of the output stage to internal stage. The location of poles in miller compensation is given as

and

$$
\mathrm{P}_{1}=\frac{1}{\mathrm{R}_{1} \mathrm{C}_{1}}
$$

$$
\mathrm{P}_{2}=\frac{1}{R_{2} C_{2}}
$$

Where $R_{1}, C_{1}$ and $R_{2}, C_{2}$ are the resistor and capacitor of stages $A_{1}$ and $A_{2}$ respectively. The RHP zero location which is generated during miller compensation is given by

$$
\mathrm{Z}_{1}=\frac{g_{m 2}}{C_{C}}
$$

This zero is to be shifted from RHP to LHP for maintaining stability by employing nulling resistor compensation. The resistor used in the proposed techniquehelps to move this zero from one plane to another plane. The location of poles in this technique is same as the miller compensation however this technique also generates one another pole which is very far away from origin and not taken into consideration.

Volume 5 Issue 6, June 2016 


\section{International Journal of Science and Research (IJSR) \\ ISSN (Online): 2319-7064}

Index Copernicus Value (2013): 6.14 | Impact Factor (2015): 6.391

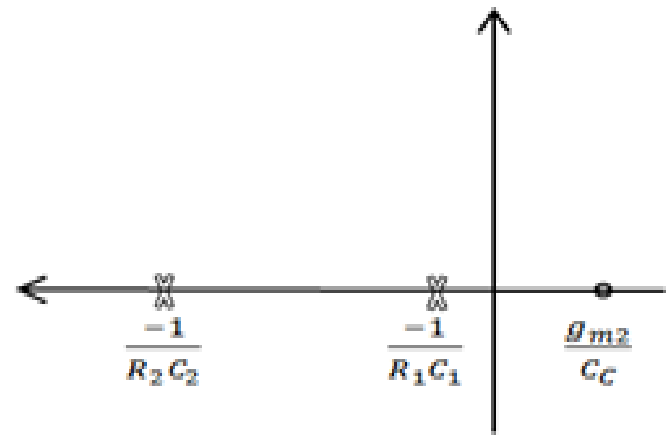

Figure 2: Location of poles and zeros

The new location of zero after employing this technique is given as

$$
Z_{1}=\frac{1}{\left(\frac{1}{g_{m 2}}-R_{Z}\right) C_{C}}
$$

Where

$\mathrm{R}_{\mathrm{z}}=$ Nulling resistor

The zero moves to infinity for the value of $R_{z}=\frac{1}{g_{m 2}}$ and for $\mathrm{R}_{\mathrm{z}}>\frac{1}{g_{m 2}}$ the zero moves from RHP to LHP and maintains the stability. This technique also improves the $3 \mathrm{~dB}$ bandwidth. The bandwidth is the reciprocal of the product of the resistor and capacitor if this resistor value is decreased the bandwidth improves.

The schematic design of Op-amp is shown in below figure which is designed by using six PMOS and five NMOS transistors including miller capacitor and resistor. In the proposed Op-amp this capacitor and resistor is designed with MOS transistor which reduces area. The transistor $\mathbf{M}_{11}$ behaves as a capacitor whereas transistor $\mathbf{M}_{10}$ is act as a resistor. For operating transistor $\mathrm{M}_{10}$ as a resistor, it is operated in triode region. In Op-amp current source used for

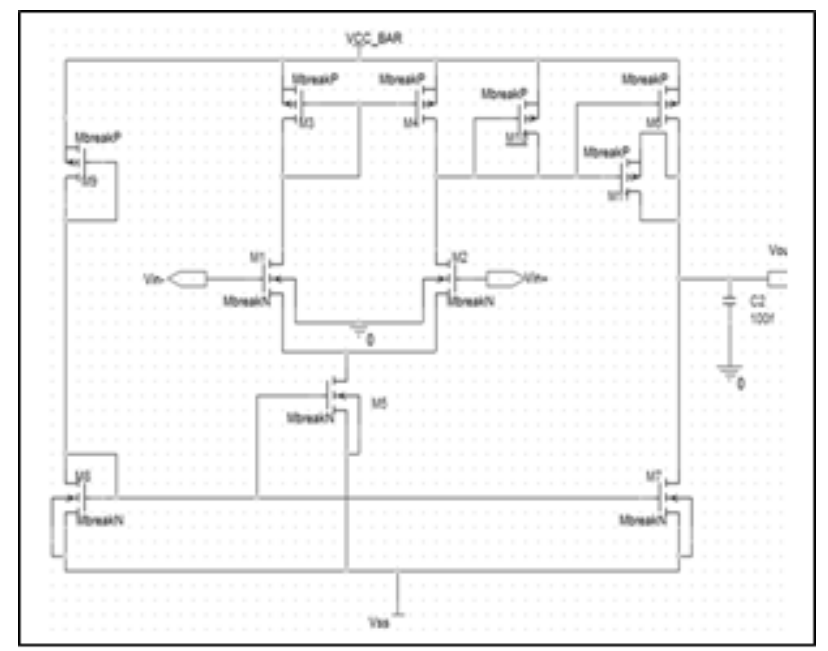

Figure 3: Design of Op-amp

maintain the transistors in particular region, which is also designed with MOS transistor. The design of Op-amp includes two basic part i.e. differential amplifier and gain stage. In this Op-amp the differential amplifier which is also referred as "Heart of Op-amp" includes transistors from $\mathrm{M}_{1}$ to $\mathrm{M}_{5}$. This amplifier includes current-mirrorconfiguration, input transistor pair and a current source at the bottom which is generally replaced with NMOS transistor. Each part is responsible for various parameters of Op-amp. The currentmirror configuration is responsible for high Input common mode range and act as a load for input stage. This first stage also provides important parameters such as Power supply rejection ratio (PSRR), Common mode rejection ratio (CMRR), high input impedance and low output impedance. The Gain-bandwidth product is depended upon input transistor pair and slew rate is given by transistor $M_{5}$. A second stage is used to improve the gain of first stage which also converts the differential input to single ended output. In the second stage, transistor $\mathrm{M}_{6}$ act as a load and provide maximum output voltage swing whereas minimum output voltage swing is provided by transistor $M_{7}$. The gain of the first stage and second stage is given as

$$
A_{V}=A_{1} A_{2}
$$

where

$$
\begin{aligned}
\mathrm{A}_{1} & =g_{m 1} R_{1} \\
\text { and } \mathrm{A}_{2} & =g_{m 2} R_{2}
\end{aligned}
$$

where $g_{m 1}$ and $g_{m 2}$ is the transconductance of stage $\mathrm{A}_{1}$ and $A_{2}$ and $R_{1}$ and $R_{2}$ is given as

$$
\begin{array}{r}
\mathrm{R}_{1}=r_{d s 2} \| r_{d s 4} \\
\text { and } \mathrm{R}_{2}=r_{d s 6} \| r_{d s 7}
\end{array}
$$

where

$r_{d s}=$ Drain to Source resistance of transistor

\section{Op-Amp Design Issue}

1) The proposed Op-amp uses Dual input single ended output differential amplifier which directly provide input to second stage. This is also referred as Dual input unbalanced output differential amplifier.

2) A load capacitance of $100 \mathrm{f} F$ is used for obtaining output with the compensation capacitor of $50 \mathrm{f} \mathrm{F}$, which is obtained from the expression

$$
C_{C} \geq 0.22 C_{L}(10)
$$

From this value of load capacitance, $C_{C}$ is given as

$$
C_{C} \geq 22 f F(11)
$$

So for this Op-amp choose the 50fF compensation capacitor which is suitable for obtaining this output.

3) Due to highly demand for low power devices for many applications this Op-amp is work with low power i.e. $1.3 \mathrm{~V}$ supply voltage. Hence there is no any issue of large power dissipation also used Vss of $-1.3 \mathrm{~V}$.

4) Overall design is simulated with only MOS transistors to reduce the area.

5) Aspect ratios of each transistor are calculated and put this value with slightly changes to obtain better response.

6) Sinusoidal input is applied at both input terminal i.e. inverting and non-inverting having magnitude of $0.5 \mathrm{~V}$ of opposite phase.

7) Op-amp parameters such as CMRR, PSRR, Slew rate, DC offset, Settling time etc. are also calculated. CMRR which is the ratio of differential mode gain to common mode gain achieve higher value due to the low value of the common mode gain.

\section{Results and Discussion}

For simulation of this Op-amp PSpice EDA tool is used with 130nm digital CMOS technology. BSIMv3.0 is used as a model library. Comparative table on previous work is shown 


\section{International Journal of Science and Research (IJSR) \\ ISSN (Online): 2319-7064}

Index Copernicus Value (2013): 6.14 | Impact Factor (2015): 6.391

in TABLE I whereas TABLE II represents simulated results of this work.

Table 1: Comparative Table of Previous Work

\begin{tabular}{|c|c|c|c|c|c|c|}
\hline Parameter & {$[\mathbf{1}]$} & {$[\mathbf{2}]$} & {$[\mathbf{3}]$} & {$[\mathbf{4}]$} & {$[\mathbf{5}]$} & {$[\mathbf{6}]$} \\
\hline Power Supply(V) & 1.8 & 1.8 & 3 & 5 & 2.5 & 3.5 \\
\hline Gain $(\mathrm{dB})$ & 65 & 58.1 & 49 & 77 & 36.74 & 48 \\
\hline $\mathrm{f}_{-3 \mathrm{~dB}}$ BW $(\mathrm{MHz})$ & - & 0.25 & - & 0.0013 & 7.33 & - \\
\hline UGB $(\mathrm{MHz})$ & 2300 & 205 & 2020 & 14 & 16.54 & 40 \\
\hline CMRR $(\mathrm{dB})$ & 96 & - & 39 & 80.9 & 133.6 & - \\
\hline PSRR $(\mathrm{dB})$ & 62 & - & 154 & - & 179.3 & - \\
\hline Slew rate $(\mathrm{V} / \mu \mathrm{s})$ & 450 & - & 1.41 & 10.3 & 12.5 & - \\
\hline DC Offset $(\mu \mathrm{W})$ & $2 \%$ & - & - & - & - & - \\
\hline Powerdiss. $(\mathrm{mW})$ & 25 & - & 0.039 & - & 0.804 & - \\
\hline Area $\left(\mathrm{mm}^{2}\right)$ & 0.04 & - & - & - & - & - \\
\hline Phase Margin & - & $84^{\circ}$ & $60^{\circ}$ & 53.4 & $48.1^{\circ}$ & $49.8^{\circ}$ \\
\hline Technology $(\mathrm{nm})$ & 180 & 500 & 180 & 350 & 180 & 180 \\
\hline
\end{tabular}

Table 2: Simulation Result Of Proposed Op-amp

\begin{tabular}{|c|c|}
\hline Parameter & Obtained Value \\
\hline Power Supply & $1.3 \mathrm{~V}$ \\
\hline Gain & $59 \mathrm{~dB}$ \\
\hline $\mathrm{f}_{-3 \mathrm{~dB}}$ Bandwidth & $1.25 \mathrm{MHz}$ \\
\hline Unity Gain Bandwidth & $605 \mathrm{MHz}$ \\
\hline CMRR & $269 \mathrm{~dB}$ \\
\hline PSRR & $106 \mathrm{~dB}$ \\
\hline Slew rate & $1.6 \mathrm{~V} / \mu \mathrm{s}$ \\
\hline DC Offset & $121 \mathrm{mV}$ \\
\hline Power dissipation & $0.43 \mathrm{~mW}$ \\
\hline Area & $125 \mu \mathrm{m}^{2}$ \\
\hline Technology & $130 \mathrm{~nm}$ \\
\hline Phase margin & $54^{\circ}$ \\
\hline Settling time & $1.46 \mu \mathrm{s}$ \\
\hline Output Resistance & $15.7 \Omega$ \\
\hline
\end{tabular}

Frequency response of this Op-amp is shown in fig. 4 where magnitude is in $\mathrm{dB}$ and frequency is in logarithmic plot as well as bode plot is shown in fig. 5 . The bode plot shows 59 $\mathrm{dB}$ gain is obtained for this Op-amp as well as phase margin of $54^{\circ}$ is achieved.

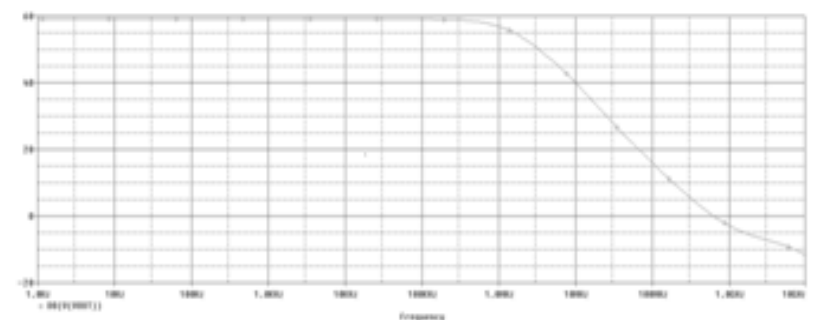

Figure 4: Frequency response of Op-amp

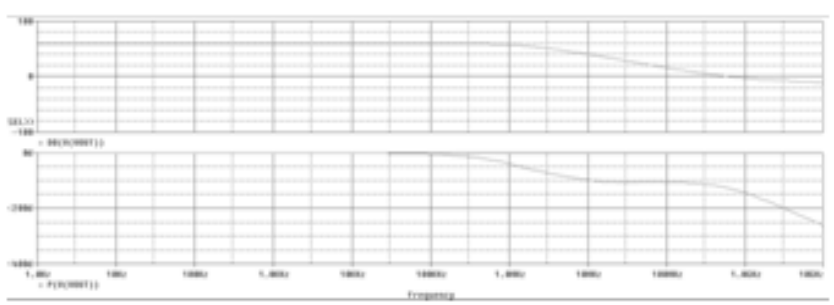

Figure 5: Bode Plot of Op-amp

\section{Acknowledgment}

With a deep sense of gratitude, the author would like to especially thank to Prof. Anil Kumar Sahu, for his helpful discussion and guidance. Also thanks to all of those who support to me for completing this research.

\section{References}

[1] Daibashish Gangopdhaya and T.K.Bhattarchyya, "A 2.3GHz gm-boosted High swing Class-AB Ultra-Wide bandwidth Operational Amplifier in 180nm CMOS," IEEE Transaction, 2010, pp. 713-716.

[2] Rishi Todani and Ashis Kumar Mal, "Design of CMOS OpAmp Using Potential Distribution Methoud," IEEE International Confrence on Circuits and Systems, October 2012.

[3] Priyanka kakotay, "Design of low voltage and high frequency CMOSoperational amplifier," International journal of VLSI design and Communiction System (VLSICS) Vol.2, March 2011

[4] Amanna yadav, "Design of two-stage Op-amp and analyze the effect of scaling," International journal of engineering reasearch and application, Vol. II, Issue 5, Sep- Oct 2012, pp.647-654

[5] Rajdeep Charterjee and Sayan Bandyopadhyay, "Design of two-stage Operational Amplifier with High CMRR and low power in 180nm Technology"International J. of Recent Trends in Enggering and Technology, vol. 12, 2014.

[6] D. Nageshwarrao and K. Suresh Kumar, "Implementation and simulation of CMOS two-stage operational amplifier,'International J. of Advance in Engineering \& Technology, Jan. 2013.

[7] P. E. Allen and Hollberg, CMOS Analog Ckt. Design, Oxford University, second edition, 2007.

[8] B.Razavi, Design of Analog CMOS Integrated Circuits, McGraw Hill Companies, 2002. 